\title{
Asymmetric induction in cyclohexadienones carrying $\alpha$-D-glucopyranosyl moiety
}

\author{
Saki Yajima ${ }^{a}$, Tsuyoshi Saitoh ${ }^{\mathrm{a}, \mathrm{b}, \mathrm{d}^{*}}$, Kohei Kawa ${ }^{\mathrm{a}}$, Kensuke Nakamura ${ }^{\mathrm{c}}$, Hiroshi Nagase ${ }^{\mathrm{b}}$, Yasuaki \\ Einaga ${ }^{\mathrm{a}, \mathrm{d}}$, and Shigeru Nishiyama ${ }^{\mathrm{a}, \mathrm{d}}$ \\ ${ }^{a}$ Department of Chemistry, Faculty of Science and Technology, Keio University, Hiyoshi 3-14-1, Kohoku-ku, Yokohama 223-8522, Japan \\ ${ }^{b}$ International Institute for Integrative Sleep Medicine, University of Tsukuba, Tennodai 1-1-1, Tsukuba 305-8575, Japan \\ ${ }^{c}$ Department of Life Science and Informatics, Maebashi Institute of Technology, Kamisadori 460-1, Maebashi 371-0816, Japan \\ ${ }^{d}$ JST CREST/ACCEL, 3-14-1 Hiyoshi, Yokohama 223-8522, Japan
}

\begin{tabular}{ll} 
ARTICLE INFO & ABSTRACT \\
\hline Article history: & $\begin{array}{l}\text { Cyclohexadienone derivatives possessing } \alpha \text {-D-glucopyranosyl moiety were designed and } \\
\text { synthesized using chemical oxidation or electrochemical oxidation with boron-doped diamond } \\
\text { Received }\end{array}$ \\
$\begin{array}{l}\text { Received in revised form } \\
\text { Accepted }\end{array}$ & $\begin{array}{l}\text { diastereoselectivity (dr ratio: }>9: 1) \text {. Conformational analyses of the } C \text {-glucoside structures were } \\
\text { performed by NOE experiments and force field calculations, and supported the observed regio- } \\
\text { svailable online }\end{array}$ \\
\hline selectivity.
\end{tabular}

Keywords:

Cyclohexadienone

2009 Elsevier Ltd. All rights reserved.

Diastereoselective hydrogenation

Hypervalent iodobenzene

Anodic oxidation

Boron-doped diamond electrodes

\section{Introduction}

Highly reactive cyclohexadienones have been synthesized by oxidation of appropriately functionalized phenol precursors, and utilized as synthetic intermediates of natural products and biologically important substances (Figure 1). In a relatively early investigation, a plausible biomimetic synthesis of asatone, a component of Asarum taitonense H., ${ }^{1}$ was achieved through spontaneous dimerization of the cyclohexadienone (type B), which was produced by anodic oxidation of the corresponding phenol (A). Closely related synthesis of penicillone $A^{2 a}$ and asatone $^{2 b}$ was reported using a chemical oxidant $\left[\mathrm{PhI}(\mathrm{OAc})_{2}\right.$ (PIDA).$^{2}$ Cationic cyclohexadienones (type C) have been generated by chemical $^{3}$ and electrochemical methods, ${ }^{1 \mathrm{~b}, 4}$ and utilized as cationic dienes in Diels-Alder reactions, leading to bicyclic derivatives, ${ }^{5}$ or as scaffolds for continuous cyclization reactions. ${ }^{6}$

We have synthesized another series of cyclohexadienones (type D) as intermediates of natural marine spiroisoxazoline products, such as aerothionins, ${ }^{7}$ calafianin, ${ }^{7}$ and fistularins. ${ }^{8}$ The absolute configurations of several members were confirmed using optically active intermediates, which were obtained successfully in pure form by diastereomeric separation. ${ }^{7}$ Acquisition of optically active cyclohexadienones has attracted a great deal of attention in connection with structural determination and biological activities. For this purpose, enantioselective oxidation of the corresponding phenols has been reported by substrate or reagent control methodologies. An example of the former was the oxidation of $O$-glycosyl catechol to the corresponding cyclohexadienone, which was automatically converted to the bicyclo[2.2.2] octanes. $^{9}$ The latter was reported by application of chiral hypervalent iodine oxidants.

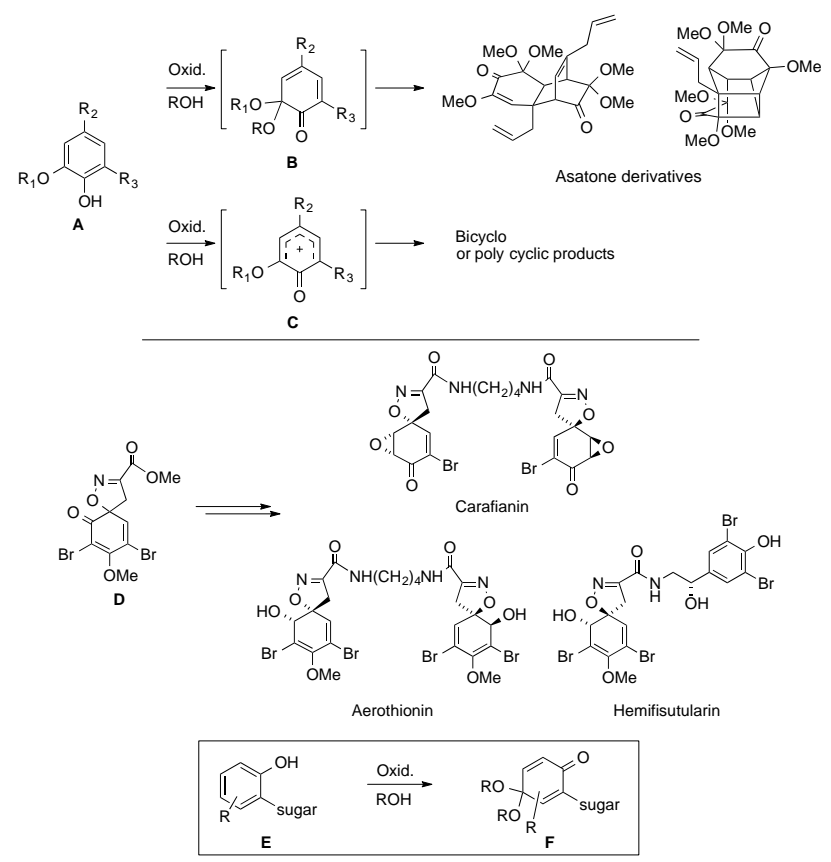

Figure 1. 2,4- and 2,5-Cyclohexadienones in natural products. 
In the present investigation, we examined the scope and limitation of the chiral induction onto cyclohexadienone

Table 2. Synthesis of $C$-glycoside 4a through $O-C$ migration.
Substrates $\mathbf{2 b}$ and $\mathbf{2 c}$ with higher electron density of the aromatic ring, provided no $C$-glycosides (4b and $\mathbf{4 c}$, entries 2, 3). Further assessment of Lewis acids revealed that TMSOTf (entry 4; ratio of $O$-glycoside vs. $C$-glycoside $=46: 31$ ) and $\mathrm{HfCl}_{4}$ (entry 5; 12:46) affected the desired conversion to afford $O$ to $C$ glycosyl migration (3a to 4a) (Table 2). ${ }^{14}$ The former case gave better material balance than the latter.

\subsection{Oxidation of glycosides}

As conversion of phenols to dienone structures proceeded by a two-electron oxidation process, chemical oxidation of the product 4a with PIDA in $\mathrm{MeOH}$ was examined. Smooth conversion by the plausible reaction process provided the desired dienone $\mathbf{5}$ in $77 \%$ yield (Scheme 1). In contrast, further attempts by anodic oxidation of $\mathbf{3 b}$ provided no expected products. In our previous work, similar difficulties had been circumvented by protection of the phenols. ${ }^{11}$ Thus, 4 a was methylated under standard reaction conditions to give the methylether 6 in $97 \%$ yield. The anodic oxidation of $\mathbf{6}$ was inspected under several reaction conditions. As outlined in Table 3, the desired reaction proceeded only when BDD electrode was used as anode (entry 3). In our previous study of electrolysis on BDD electrodes, we reported the generation of methoxyradicals on BDD electrodes better than the others and methoxyradicals mediated the anodic oxidation of dimethoxybenzene. ${ }^{15}$ This observation suggested that the oxidative methoxylation and followed oxidation reactions would be mediated by methoxyradicals. In addition, in the flow cell system which manipulate methoxy radicals, 5 was provided in $49 \%$ yield along with the recovered educt 6 (entry 4 ).

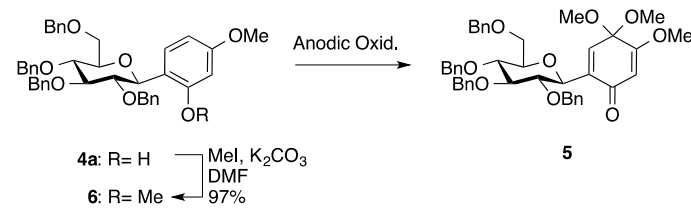

Scheme 1. Phenol oxidation into 2,5-cyclohexadienone.

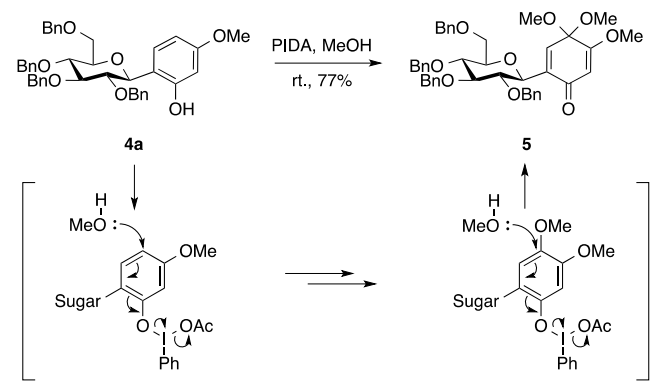

Table 3. Anodic oxidation of $C$-glycoside $6^{\mathrm{a}}$.

\begin{tabular}{|c|c|c|c|}
\hline $\begin{array}{r}\mathrm{BnO} \\
\mathrm{BnO} \\
\mathrm{BnO}\end{array}$ & $I^{\mathrm{OMe}}$ & $\underset{30^{\circ} \mathrm{C} \text { to rt. }}{\text { idd, } \mathrm{MS} 4 \mathrm{~A}}$ & \\
\hline \multirow{2}{*}{ Entries } & \multirow{2}{*}{ Lewis Acid } & \multicolumn{2}{|c|}{ Yield (\%) } \\
\hline & & $O$-Glycoside $\mathbf{3 a}$ & $C$-Glycoside $\mathbf{4 a}$ \\
\hline 1 & $\mathrm{ZrCl}_{4}$ (>10 eq.) & \multicolumn{2}{|c|}{ no reaction } \\
\hline 2 & $\mathrm{Sc}(\mathrm{OTf})_{3}(>10$ eq. $)$ & \multicolumn{2}{|c|}{ no reaction } \\
\hline 3 & $\mathrm{Yb}(\mathrm{OTf})_{3}(>10$ eq. $)$ & \multicolumn{2}{|c|}{ no reaction } \\
\hline 4 & $\mathrm{HfCl}_{4}$ (1 eq.) & 12 & 46 \\
\hline
\end{tabular}




\subsection{Catalytic hydrogenation}

During manipulation of the cyclohexadienone attached to the glycosyl moiety 5, Pd/C-catalyzed hydrogenation was found to

Table 4. Hydrogenation of $C$-glycosides 5, 9-11. ${ }^{\text {a }}$

\begin{tabular}{|c|c|c|c|c|}
\hline \multicolumn{2}{|c|}{$5,9,10,11$} & \multirow{2}{*}{\multicolumn{2}{|c|}{$\begin{array}{l}\text { 7a, 12a, 13a, 14a } \\
\text { Yields }(\%)^{b} \\
\end{array}$}} & $7 b, 12 b, 13 b, 14 b$ \\
\hline Entries & Educts (R) & & & $d r(\mathbf{a}: \mathbf{b})$ \\
\hline 1 & $5(\mathrm{Bn})$ & $7 \mathbf{a}(15)$ & $7 \mathbf{b}(16)$ & $42: 58$ \\
\hline 2 & 9 (Ac) & 12a (49) & $12 b(14)$ & $78: 22$ \\
\hline 3 & $10(\mathrm{Bz})$ & 13a (75) & $13 b(8)$ & $90: 10$ \\
\hline 4 & 11 (Boc) & $14 a(30)$ & 14b (11) & $73: 27$ \\
\hline
\end{tabular}
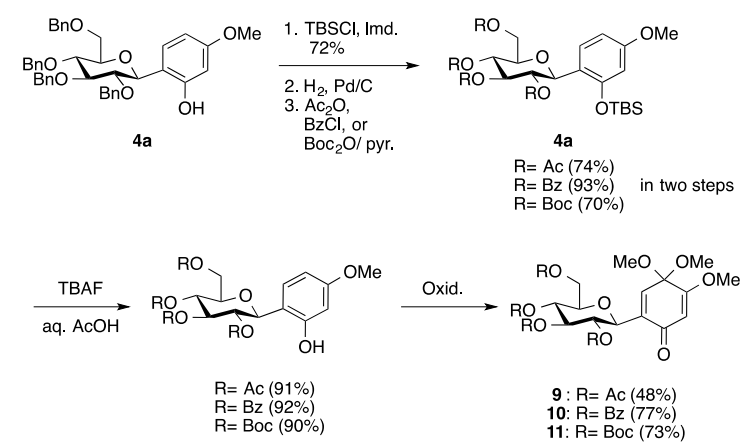

Scheme 3. Synthesis of $\mathbf{9 - 1 0 .}$

Table 5. Effects of solvents on diastereoselective hydrogenation. ${ }^{a}$

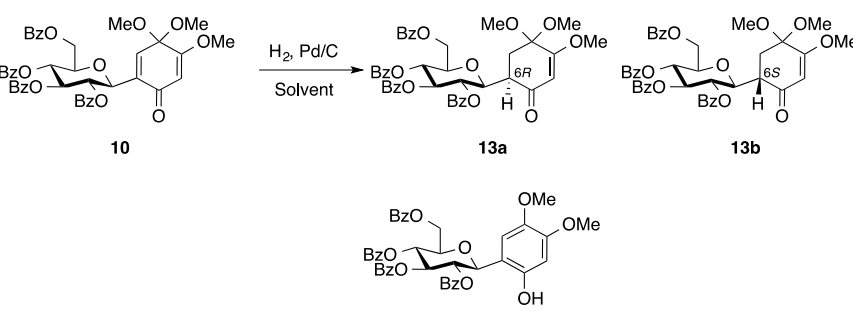

\begin{tabular}{ccccccc}
\hline & \multicolumn{7}{c}{ Yields $(\%)^{\mathrm{b}}$} & \multirow{2}{c}{$\begin{array}{c}\text { dr ratio } \\
\text { Entries }\end{array}$} & Solvent & $\mathbf{1 0}$ & $\mathbf{1 3 a}$ & $\mathbf{1 3 b}$ & $\mathbf{1 5}$ & \\
\cline { 3 - 6 } 1 & dioxane & 2.3 & 59 & 7 & n.d. & $90: 10$ \\
2 & THF & 7.5 & 65 & 6 & n.d. & $92: 8$ \\
3 & tBuOH & 12.5 & n.d. & n.d. & 40 & n.d. \\
4 & acetone & 2.1 & 75 & 8 & 1 & $90: 10$ \\
5 & EtOH & 24.3 & 23 & 0.2 & 49 & $99: 1$ \\
6 & DMF & 38 & 43 & 3 & 16 & $93: 7$ \\
7 & MeOH/EtOAc & n.d. & 59 & 4 & n.d. & $93: 7$ \\
\hline
\end{tabular}

${ }^{a}$ Reaction conditions: cyclohexadienone in acetone $(0.05 \mathrm{M}), 5 \% \mathrm{Pd} / \mathrm{C}(10$ $\mathrm{mol} \%), \mathrm{H}_{2}$ atmosphere, room temperature.

${ }^{\mathrm{b}}$ Isolated yield.
In the hydrogenation reactions of $\mathbf{1 0}$, protic solvents facilitated the production of the aromatic compound $\mathbf{1 5}$ as main products (entries 3 and 5 in Table 4), while aprotic solvents suppressed generation of $\mathbf{1 5}$. This is probably because protic solvent allows proton migration to generate the corresponding arylic system. The highest regioselectivity was achieved in acetone as solvent. Interestingly, the preferential 13a-type diastereoselectivity was observed in all of the solvents we used. Structures of the products were confirmed by NOE experiments. In 13a, C-8 proton with a cis-relationship to the C-7 proton was correlated with the C-2 proton of the glycosyl moiety. Similarly, 13b showed a correlation between C-8 carrying trans-relationship to C-7 and C2 (Figure 2). To support the NMR-based structural elucidation, lowest energy conformations of 12a and 12b were calculated by MOE (Molecular Operating Environment, ver. moe 2012. 1001) using the Born solvation model with the dielectric constant of $\mathrm{CHCl}_{3}$ (4.8). As indicated in Figure 2, the configurations at C-7 in 12a and 12b were in good accordance with those obtained by the NOE measurement. The same C-7 configurations in $\mathbf{7 a}, \mathbf{b}$, 13a,b, and 14a,b as 12a,b were confirmed by chemical conversion of the protecting groups. Although the $6 R$ configuration in the hydrogenated derivatives was dominantly produced in the acyl derivatives $(\mathbf{1 2}, \mathbf{1 3}$, and 14), the benzyl derivative 7 showed the opposite stereoselectivity. To confirm the reaction prospect, theoretical calculations were carried out to estimate the opposite stereoselectivity. To confirm the reaction prospect, theoretical calculations were carried out to estimate the conformational abundance ratio in solution. Conformers of compounds 5, 9, 10, and 11 were corrected with the following conditions. Stability of each conformers are within $2.75 \mathrm{kcal} / \mathrm{mol}$ from the global minimum, and each structures are at least 0.015 angstrom root mean square distance (RMSD) apart from each other. The diastereomer ratio predicted by the calculation agreed quite well with the experimental data (Table 5). This observation indicated that the theoretical calculation can reproduce the remarkable effect of the substrate conformations in solutions.

Figure 2. Structures of $\mathbf{1 2 a}$ and $\mathbf{1 2 b}$ and NOE correlations.
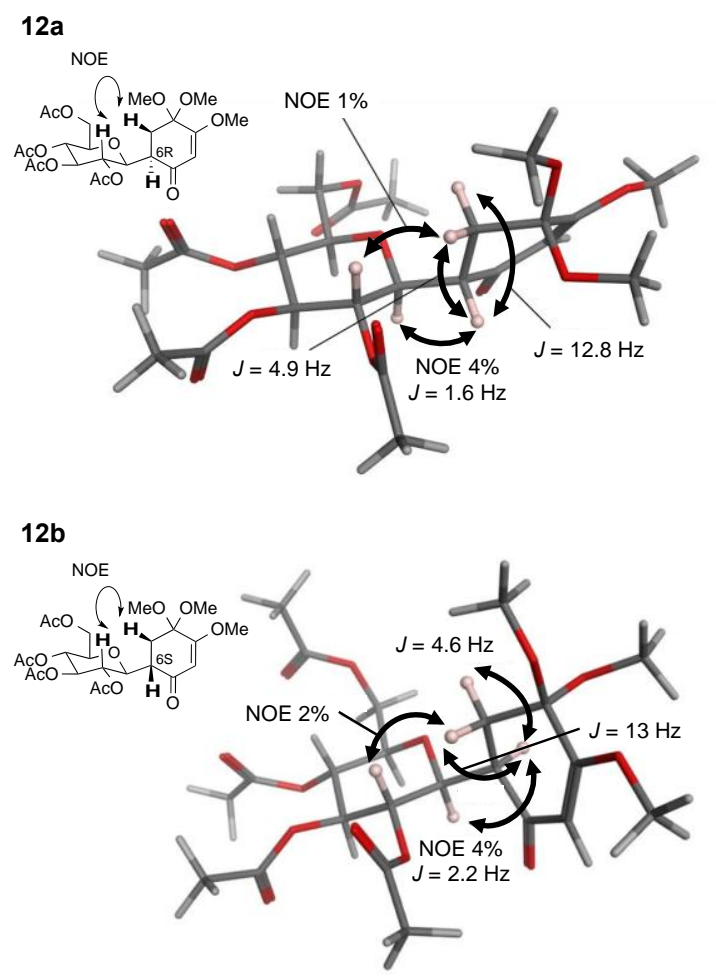
Table 5. Conformation of $C$-glycosides and comparison of selectivity.

1

2

3

4

5

6

7

8
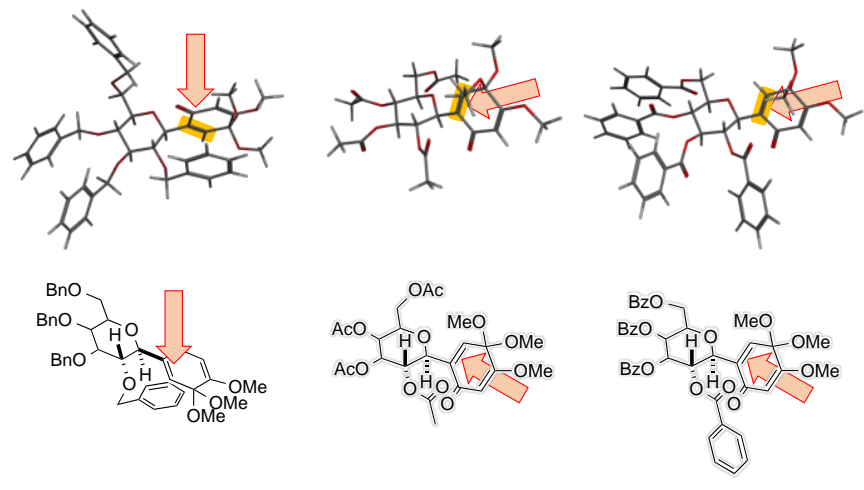

9

\begin{tabular}{ccc}
\hline \multirow{2}{*}{ Comp. } & \multicolumn{2}{c}{$d r$ ratio } \\
& Experimental & Calculated \\
\hline $\mathbf{5}$ & $42: 58$ & $11: 89$ \\
$\mathbf{9}$ & $78: 22$ & $90: 10$ \\
$\mathbf{1 0}$ & $90: 10$ & $92: 8$ \\
$\mathbf{1 1}$ & $73: 27$ & $64: 36$ \\
\hline
\end{tabular}

\section{Conclusion}

In conclusion, cyclohexadienone derivatives carrying the $\alpha$-Dglucopyranosyl moiety were designed and synthesized using chemical or anodic oxidation in the final oxidative stage. In the chiral induction reactions, catalytic hydrogenation provided high diastereoselectivity, although low yields and stereoselectivity were observed in 1,2-nucleophilic addition reaction. ${ }^{16,17}$ The conformations of the $C$-glucoside structures responsible for the selectivity were examined by NOE experiments and force field calculations.

\section{Experimental section \\ 4.1. General information}

All reactions were carried out under an argon atmosphere unless otherwise noted. Dry THF, dry $\mathrm{Et}_{2} \mathrm{O}$ and dry $\mathrm{CH}_{2} \mathrm{Cl}_{2}$ were purchased from Kanto Chemical Co., Inc. Optical rotations were measured on a JASCO P-2200 digital polarimeter with a sodium (D line) lamp. IR spectra were recorded on a JASCO Model A202 spectrophotometer. ${ }^{1} \mathrm{H}-\mathrm{NMR}$ spectra and ${ }^{13} \mathrm{C}-\mathrm{NMR}$ spectra were obtained on JNM- $\alpha 400$, JNM-AL400 and JNM-ECX400 spectrometers in deuterated solvent. Deuteriochloroform was used as a solvent, unless otherwise stated. High-resolution mass spectra were obtained on Waters LCT Piemier XE (ESI). Preparative and analytical TLC were carried out on silica gel plate (Kieselgel 60 F254, E. Merck AG., Germany) using UV light and/or 5\% molybdophosphoric acid in ethanol for detection. Kanto silica $60 \mathrm{~N}$ (spherical, neutral, 105-210 $\mu \mathrm{M}$ ) was used for column chromatography.

4.1.1. (2R, 3R, 4S, 5R)-3,4,5-Tris(benzyloxy)-2((benzyloxy)methyl)-6-(3methoxyphenoxy) tetrahydro-2H-pyran (3a) and 5methoxy-2-((2S, 3S, 4R, 5R,6R)-3,4,5-tris (benzyloxy)6-((benzyloxy)methyl)tetrahydro-2H-pyran-2yl)phenol (4a).

To a stirring mixture of $\mathbf{1}^{12}(200 \mathrm{mg}, 0.29 \mathrm{mmol})$, 3methoxyphenol (2a, $0.025 \mathrm{~mL}, 0.8 \mathrm{mmol})$, and MS4A $(150 \mathrm{mg})$ in anhydrous $\mathrm{CH}_{2} \mathrm{Cl}_{2}(1 \mathrm{~mL})$ was added TMSOTf $(4 \mathrm{~mL}, 0.03$ $\mathrm{mmol})$ in $\mathrm{CH}_{2} \mathrm{Cl}_{2}(0.5 \mathrm{~mL})$. After being stirred at $-30{ }^{\circ} \mathrm{C}$ for 30 min, the reaction temperature was raised to room temperature. After $15 \mathrm{~h}$, the reaction was quenched by the addition of sat. aq. $\mathrm{NaHCO}_{3}$. The resulting mixture was filtered through a Celite pad. The filtrate was washed with sat. aq. $\mathrm{NaHCO}_{3}$, and brine, dried $\left(\mathrm{Na}_{2} \mathrm{SO}_{4}\right)$. After concentration in vacuo, the residue was purified by silica gel column chromatography $\left(\mathrm{CHCl}_{3}\right.$ : hexane : EtOAc $=$ $4 / 8 / 1)$ to afford $\mathbf{3 a}^{17}$ as a colorless oil $(109 \mathrm{mg}, 73 \%, \sim 1: 1$ mixture) and $\mathbf{4 a} \mathbf{a}^{12 \mathrm{~b}}$ as a colorless oil $(24.0 \mathrm{mg}, 16 \%)$, respectively. 3a ( $\alpha$-isomer): ${ }^{1} \mathrm{H}$ NMR (400 $\left.\mathrm{MHz}, \mathrm{CDCl}_{3}\right) \delta$ 7.39-7.08 (m, $20 \mathrm{H}), 7.16(\mathrm{dd}, J=8.3,8.3 \mathrm{~Hz}, 1 \mathrm{H}), 6.69(\mathrm{dd}, J=8.3,2.3 \mathrm{~Hz}$, $1 \mathrm{H}), 6.66(\mathrm{dd}, J=2.5,2.3 \mathrm{~Hz}, 1 \mathrm{H}), 6.58(\mathrm{dd}, J=8.3,2.5 \mathrm{~Hz}$, $1 \mathrm{H}), 5.46(\mathrm{~d}, J=3.6 \mathrm{~Hz}, 1 \mathrm{H}), 5.05(\mathrm{~d}, J=10.6 \mathrm{~Hz}, 1 \mathrm{H}), 4.88(\mathrm{~d}$, $J=11.2 \mathrm{~Hz}, 1 \mathrm{H}), 4.86(\mathrm{~d}, J=11.2 \mathrm{~Hz}, 1 \mathrm{H}), 4.79(\mathrm{~d}, J=12.1 \mathrm{~Hz}$, $1 \mathrm{H}), 4.68(\mathrm{~d}, J=12.1 \mathrm{~Hz}, 1 \mathrm{H}), 4.59(\mathrm{~d}, J=12.0 \mathrm{~Hz}, 1 \mathrm{H}), 4.49(\mathrm{~d}$, $J=10.6 \mathrm{~Hz}, 1 \mathrm{H}), 4.41(\mathrm{~d}, J=12.0 \mathrm{~Hz}, 1 \mathrm{H}), 4.19(\mathrm{dd}, J=9.2,9$. $\mathrm{Hz}, 1 \mathrm{H}$ ), 3.87 (ddd, $J=10.3,3.0,2.0 \mathrm{~Hz}, 1 \mathrm{H}$ ), 3.78 (dd, $J=10.3$, $9.2 \mathrm{~Hz}, 1 \mathrm{H}), 3.75(\mathrm{~s}, 3 \mathrm{H}), 3.7249(\mathrm{dd}, J=10.8,2.5 \mathrm{~Hz}, 1 \mathrm{H}), 3.72$ $(\mathrm{dd}, J=9.2,3.0 \mathrm{~Hz}, 1 \mathrm{H}), 3.57$ (dd, $J=10.8,2.0 \mathrm{~Hz}, 1 \mathrm{H}) .3 \mathrm{a}(\beta-$ isomer): ${ }^{1} \mathrm{H}$ NMR (400 MHz, $\left.\mathrm{CDCl}_{3}\right) \delta 7.39-7.08(\mathrm{~m}, 20 \mathrm{H}), 7.16$ $(\mathrm{dd}, J=8.3,8.3 \mathrm{~Hz}, 1 \mathrm{H}), 6.69(\mathrm{dd}, J=8.3,2.3 \mathrm{~Hz}, 1 \mathrm{H}), 6.66$ (dd, $J=2.5,2.3 \mathrm{~Hz}, 1 \mathrm{H}), 6.58(\mathrm{dd}, J=8.3,2.5 \mathrm{~Hz}, 1 \mathrm{H}), 5.00(\mathrm{~d}, J=$ $3.6 \mathrm{~Hz}, 1 \mathrm{H}), 5.05(\mathrm{~d}, J=10.6 \mathrm{~Hz}, 1 \mathrm{H}), 4.88(\mathrm{~d}, J=11.2 \mathrm{~Hz}, 1 \mathrm{H})$, $4.86(\mathrm{~d}, J=11.2 \mathrm{~Hz}, 1 \mathrm{H}), 4.79(\mathrm{~d}, J=12.1 \mathrm{~Hz}, 1 \mathrm{H}), 4.68(\mathrm{~d}, J=$ $12.1 \mathrm{~Hz}, 1 \mathrm{H}), 4.59(\mathrm{~d}, J=12.0 \mathrm{~Hz}, 1 \mathrm{H}), 4.49(\mathrm{~d}, J=10.6 \mathrm{~Hz}$, $1 \mathrm{H}), 4.41(\mathrm{~d}, J=12.0 \mathrm{~Hz}, 1 \mathrm{H}), 4.19(\mathrm{dd}, J=9.2 \mathrm{~Hz}, 9.2 \mathrm{~Hz}, 1 \mathrm{H})$, 3.87 (ddd, $J=10.3,3.0,2.0 \mathrm{~Hz}, 1 \mathrm{H}), 3.78$ (dd, $J=10.3,9.2 \mathrm{~Hz}$, $1 \mathrm{H}), 3.72$ (s, 3H), $3.72(\mathrm{dd}, J=10.8,2.5 \mathrm{~Hz}, 1 \mathrm{H}), 3.72(\mathrm{dd}, J=$ 9.2, $3.0 \mathrm{~Hz}, 1 \mathrm{H}), 3.57(\mathrm{dd}, J=10.8,2.0 \mathrm{~Hz}, 1 \mathrm{H}) .4 \mathrm{a}:{ }^{1} \mathrm{H} \mathrm{NMR}$ $\left(400 \mathrm{MHz}, \mathrm{CDCl}_{3}\right) \delta 7.84(\mathrm{~s}, 1 \mathrm{H}), 7.01-7.34(\mathrm{~m}, 20 \mathrm{H}), 7.06(\mathrm{~d}, J$ $=8.3 \mathrm{~Hz}, 1 \mathrm{H}), 6.52(\mathrm{~d}, J=2.7 \mathrm{~Hz}, 1 \mathrm{H}), 6.47(\mathrm{dd}, J=2.7,8.4 \mathrm{~Hz}$, $1 \mathrm{H}), 4.98-4.37(\mathrm{~m}, 8 \mathrm{H}), 3.93-3.67(\mathrm{~m}, 6 \mathrm{H}), 3.77(\mathrm{~s}, 3 \mathrm{H}), 3.57$ (d, $J=9.6 \mathrm{~Hz}, 1 \mathrm{H})$.

\subsection{2. (2R,3R,4S,5R)-3,4,5-Tris(benzyloxy)-2- ((benzyloxy)methyl)-6-(2,3- \\ dimethoxyphenoxy)tetrahy dro-2H-pyran ( $3 b)$.}

A mixture of 1 (50.0 mg, $0.0732 \mathrm{mmol})$, 2,3-dimethoxyphenol (2b, $18 \mu \mathrm{L}, 0.138 \mathrm{mmol})$ and MS4A (100 mg) in anhydrous $\mathrm{CH}_{2} \mathrm{Cl}_{2}(0.5 \mathrm{~mL})$ was treated with TMSOTf $(10 \mu \mathrm{L}, 0.0769$ $\mathrm{mmol})$ in $\mathrm{CH}_{2} \mathrm{Cl}_{2}(0.5 \mathrm{~mL})$, essentially by the same procedure as the case of $\mathbf{3 a}$ and $\mathbf{4 a}$, to afford $\mathbf{3 b}$ as a colorless oil $(44.6 \mathrm{mg}$, $90 \%, \alpha / \beta$ ratio $=2: 1)$. Chromatographic separation provided both of the glycosides in pure form. $3 \mathbf{b}$ ( $\beta$-isomer): $[\alpha]_{\mathrm{D}}{ }^{21}-7.4$ (c 1.0, $\mathrm{CHCl}_{3}$ ); IR (film) 2863, $1086 \mathrm{~cm}^{-1}$; ${ }^{1} \mathrm{H}$ NMR (400 MHz, $\mathrm{CDCl}_{3}$ ) $\delta 7.33-7.25(\mathrm{~m}, 18 \mathrm{H}), 7.11(\mathrm{dd}, J=7.3,2.0 \mathrm{~Hz}, 2 \mathrm{H}), 6.88(\mathrm{t}, J=$ $8.5 \mathrm{~Hz}, 1 \mathrm{H}), 6.72(\mathrm{dd}, J=8.5,1.2 \mathrm{~Hz}, 1 \mathrm{H}), 6.60(\mathrm{dd}, J=8.5,1.2$ $\mathrm{Hz}, 1 \mathrm{H}), 5.49$ (d, $J=3.4 \mathrm{~Hz}, 1 \mathrm{H}), 4.99(\mathrm{~d}, J=10.7 \mathrm{~Hz}, 1 \mathrm{H}), 4.84$ $(\mathrm{d}, J=10.7 \mathrm{~Hz}, 1 \mathrm{H}), 4.82(\mathrm{~d}, J=10.7 \mathrm{~Hz}, 1 \mathrm{H}), 4.73(\mathrm{~s}, 2 \mathrm{H}), 4.54$ $(\mathrm{d}, J=12.2 \mathrm{~Hz}, 1 \mathrm{H}), 4.46(\mathrm{~d}, J=10.7 \mathrm{~Hz}, 1 \mathrm{H}), 4.36(\mathrm{~d}, J=12.2$ $\mathrm{Hz}, 1 \mathrm{H}), 4.18(\mathrm{t}, J=9.3 \mathrm{~Hz}, 1 \mathrm{H}), 4.01(\mathrm{dt}, J=10.2,2.3 \mathrm{~Hz}, 1 \mathrm{H})$, $3.83(\mathrm{~s}, 3 \mathrm{H}), 3.81(\mathrm{~s}, 3 \mathrm{H}), 3.75-3.67(\mathrm{~m}, 4 \mathrm{H}), 3.56(\mathrm{dd}, J=10.7$, $2.0 \mathrm{~Hz}, 1 \mathrm{H}) ;{ }^{13} \mathrm{C}$ NMR $\left(100 \mathrm{MHz}, \mathrm{CDCl}_{3}\right) \delta 153.7,150.9,140.2$, 138.9, 138.4, 138.3, 138.0, 128.52, 128.49, 128.47, 128.2, 128.1, $128.0,127.93,127.85,127.8,127.7,123.6,111.3,107.1,97.3$, 81.9, 80.0, 77.6, 75.8, 75.2, 73.6, 73.1, 68.4, 61.3, 56.2. HRMS (ESI) calcd for $\mathrm{C}_{42} \mathrm{H}_{44} \mathrm{O}_{8} \mathrm{Na}, 699.2934(\mathrm{M}+\mathrm{Na})$, found $\mathrm{m} / z$ 699.2936. 3b ( $\alpha$-isomer): $[\alpha]_{\mathrm{D}}{ }^{22}+82.3\left(c 1.0, \mathrm{CHCl}_{3}\right)$; IR (film) 2937, $1086 \mathrm{~cm}^{-1}$; ${ }^{1} \mathrm{H}$ NMR $\left(400 \mathrm{MHz}, \mathrm{CDCl}_{3}\right) \delta$ 7.40-7.17 (m, $20 \mathrm{H}), 6.95(\mathrm{t}, J=8.4 \mathrm{~Hz}, 1 \mathrm{H}), 6.85(\mathrm{dd}, J=8.4,1.3 \mathrm{~Hz}, 1 \mathrm{H})$, $6.66(\mathrm{dd}, J=8.4,1.3 \mathrm{~Hz}, 1 \mathrm{H}), 5.21(\mathrm{~d}, J=10.7 \mathrm{~Hz}, 1 \mathrm{H}), 4.99(\mathrm{~d}$, $J=11 \mathrm{~Hz}, 1 \mathrm{H}), 4.99(\mathrm{~d}, J=11 \mathrm{~Hz}, 1 \mathrm{H}), 4.86(\mathrm{~d}, J=10.7 \mathrm{~Hz}$, $1 \mathrm{H}), 4.85(\mathrm{~d}, J=10 \mathrm{~Hz}, 1 \mathrm{H}), 4.83(\mathrm{~d}, J=10.7 \mathrm{~Hz}, 1 \mathrm{H}), 4.59(\mathrm{~d}, J$ $=10 \mathrm{~Hz}, 1 \mathrm{H}), 4.57(\mathrm{~d}, J=10.7 \mathrm{~Hz}, 1 \mathrm{H}), 4.53(\mathrm{~d}, J=12.0 \mathrm{~Hz}$, $1 \mathrm{H}), 3.88(\mathrm{~s}, 3 \mathrm{H}), 3.86(\mathrm{~s}, 3 \mathrm{H}), 3.80-3.62(\mathrm{~m}, 6 \mathrm{H}) ;{ }^{13} \mathrm{C} \mathrm{NMR}$ $\left(100 \mathrm{MHz}, \mathrm{CDCl}_{3}\right) \delta 153.8,153.3,151.6,139.3,138.7,138.4$, 138.2 , 138.1, 128.6, 128.5, 128.5, 128.1, 128.0, 127.9, 127.8, 
$127.7,123.9,110.1,106.9,102.5,84.8,81.9,77.8,75.9,75.2$, 74.9, 73.6, 69.0, 61.1, 56.2, 55.0. HRMS (ESI) calcd for $\mathrm{C}_{42} \mathrm{H}_{44} \mathrm{O}_{8} \mathrm{Na}$, $699.2934(\mathrm{M}+\mathrm{Na})$, found $\mathrm{m} / \mathrm{z} 699.2910$.
4.2.3. 4,4,5-Trimethoxy-2-((2S,3S,4R,5R,6R)3,4,5-tris(benzyloxy)-6-

((benzyloxy)methyl)tetrahydro-2H-pyran-2yl)cyclohexa-2,5-dien-1-one (5) via anodic oxidation.

A solution of $6(20.0 \mathrm{mg}, 0.0303 \mathrm{mmol})$ in $\mathrm{MeOH}(3 \mathrm{~mL})$ containing $\mathrm{KOH}(47.0 \mathrm{mg})$ as a supporting salt was electrolyzed under constant current electrolysis conditions using BDD, platinum or glassy carbon as an anode and platinum wire or plate as a cathode. The reaction mixture was diluted with EtOAc and washed with $\mathrm{H}_{2} \mathrm{O}$. The organic layer was washed with brine, dried $\left(\mathrm{Na}_{2} \mathrm{SO}_{4}\right)$, and concentrated in vacuo. The residue was purified by PTLC (hexane : EtOAc $=2 / 1$, two times developped ) to afford $\mathbf{5}$ as a yellow oil.

\subsection{Synthesis of Ac (9), Bz (10), and Boc (11) protected C-} glycosides

4.3.1. (2R, 3R, 4R, 5S, 6S)-2-(Acetoxymethyl)-6(3,3,4-trimethoxy-6-oxocyclohexa-1,4-dien-1yl)tetrahydro-2H-pyran-3,4,5-triyl triacetate (9).

To a solution of $4 \mathbf{a}(336 \mathrm{mg}, 0.520 \mathrm{mmol})$ in $\mathrm{CH}_{2} \mathrm{Cl}_{2}(5 \mathrm{~mL})$ were added $\mathrm{TBSCl}(235 \mathrm{mg}, 1.56 \mathrm{mmol})$ and imidazole $(106 \mathrm{mg}$, $1.56 \mathrm{mmol}$ ) at $0{ }^{\circ} \mathrm{C}$. After being stirred at room temperature for 2 $\mathrm{h}$, the reaction was quenched by the addition of sat. aq. $\mathrm{NaHCO}_{3}$, and extracted with $\mathrm{CHCl}_{3}$. The organic layer was washed with brine, dried $\left(\mathrm{Na}_{2} \mathrm{SO}_{4}\right)$. After concentration in vacuo, the residue was purified by silica gel column chromatography $\left(\mathrm{CHCl}_{3}\right.$ : hexane : EtOAc $=4 / 1 / 1)$ to afford a siloxy ether $(286 \mathrm{mg}, 72 \%)$ : $[\alpha]_{\mathrm{D}}{ }^{21}+20.8\left(c 1.00, \mathrm{CHCl}_{3}\right)$; IR (film) $2858,1065 \mathrm{~cm}^{-1}$; ${ }^{1} \mathrm{H} \mathrm{NMR}$ $\left(400 \mathrm{MHz}, \mathrm{CDCl}_{3}\right) \delta 7.37-7.17(\mathrm{~m}, 20 \mathrm{H}), 7.00(\mathrm{~d}, J=3.6 \mathrm{~Hz}$, $1 \mathrm{H}), 6.55(\mathrm{dd}, J=8.8,2.0 \mathrm{~Hz}, 1 \mathrm{H}), 6.36(\mathrm{~d}, J=2.0 \mathrm{~Hz}, 1 \mathrm{H}), 4.94$ $(\mathrm{d}, J=11.1 \mathrm{~Hz}, 1 \mathrm{H}), 4.89(\mathrm{~d}, J=10.3 \mathrm{~Hz}, 1 \mathrm{H}), 4.87(\mathrm{~d}, J=11.1$ $\mathrm{Hz}, 1 \mathrm{H}), 4.75$ (br s, 1H), 4.65 (d, $J=12.5 \mathrm{~Hz}, 1 \mathrm{H}), 4.64$ (d, $J=$ $10.3 \mathrm{~Hz}, 1 \mathrm{H}), 4.53(\mathrm{~d}, J=12.5 \mathrm{~Hz}, 1 \mathrm{H}), 4.40(\mathrm{~d}, J=11.0 \mathrm{~Hz}$, $1 \mathrm{H}), 4.06(\mathrm{~d}, J=11.0 \mathrm{~Hz}, 1 \mathrm{H}), 3.78(\mathrm{~s}, 3 \mathrm{H}$, overlapped with $4 \mathrm{H}$ signals), 3.68-3.56 (m, 2H), 0.99 (s, 9H), 0.21 (s, 3H), $0.20(\mathrm{~s}$, $3 \mathrm{H}) ;{ }^{13} \mathrm{C} \mathrm{NMR}\left(100 \mathrm{MHz}, \mathrm{CDCl}_{3}\right) \delta 160.2,154.9,138.9,138.6$, $138.5,138.4,128.53,128.45,128.42,128.38,128.2,128.1$, $127.9,127.8,127.60,127.58,127.3,122.6,106.5,105.2,87.2$, 84.0, 79.6, 78.5, 77.5, 77.4, 77.2, 76.8, 75.7, 75.3, 74.4, 73.5, 69.3, 55.3, 25.9, 18.4, -4.2, -4.4. HRMS (ESI) calcd for $\mathrm{C}_{47} \mathrm{H}_{56} \mathrm{O}_{7} \mathrm{NaSi}$, $783.3693(\mathrm{M}+\mathrm{Na})$, found $\mathrm{m} / \mathrm{z} 783.3677$.

To a solution of the siloxy ether $(130 \mathrm{mg}, 0.171 \mathrm{mmol})$ in $\mathrm{MeOH}(2.5 \mathrm{~mL})$ was added 5\% Pd-C (10 mol \%). After being stirred under $\mathrm{H}_{2}$ atmosphere at room temperature for $2 \mathrm{~h}$, the reaction mixture was filtered and the filtrate was concentrated in vacuo. To a solution of the resulting residue in pyridine $(1.0 \mathrm{~mL})$ was added $\mathrm{Ac}_{2} \mathrm{O}(1.0 \mathrm{~mL})$ at $0{ }^{\circ} \mathrm{C}$. After $3 \mathrm{~h}$, the reaction was quenched by sat. aq. $\mathrm{NH}_{4} \mathrm{Cl}$ and extracted with EtOAc. The organic layer was washed with brine, dried $\left(\mathrm{Na}_{2} \mathrm{SO}_{4}\right)$. The filtrate was concentrated in vacuo., and the residue was purified by silica gel column chromatography (hexane : EtOAc $=2 / 1$ ) to afford an acetyl derivative as a colorless oil $(71.9 \mathrm{mg}, 74 \%$ yield $):[\alpha]_{\mathrm{D}}{ }^{23}-$ 2.2 (c 1.00, $\mathrm{CHCl}_{3}$ ); IR (film) $1757 \mathrm{~cm}^{-1} ;{ }^{1} \mathrm{H}$ NMR $(400 \mathrm{MHz}$, $\left.\mathrm{CDCl}_{3}\right) \delta 7.27(\mathrm{~d}, J=8.8 \mathrm{~Hz}, 1 \mathrm{H}), 6.52(\mathrm{dd}, J=8.8,2.4 \mathrm{~Hz}, 1 \mathrm{H})$, $6.33(\mathrm{~d}, J=2.4 \mathrm{~Hz}, 1 \mathrm{H}), 5.35(\mathrm{t}, J=9.5 \mathrm{~Hz}, 1 \mathrm{H}), 5.28(\mathrm{t}, J=9.5$ $\mathrm{Hz}, 1 \mathrm{H}), 5.20(\mathrm{t}, J=9.5 \mathrm{~Hz}, 1 \mathrm{H}), 4.85(\mathrm{~d}, J=9.5 \mathrm{~Hz}, 1 \mathrm{H}), 4.24$ (dd, $J=12.3,4.7 \mathrm{~Hz}, 1 \mathrm{H}), 4.09$ (dd, $J=12.3,2.1 \mathrm{~Hz}, 1 \mathrm{H}), 3.76$ (ddd, $J=9.5,4.7,2.1 \mathrm{~Hz}, 1 \mathrm{H}), 3.75(\mathrm{~s}, 3 \mathrm{H}), 2.051$ (s, 3H), 2.047 (s, 3H), 2.01 (s, 3H), 1.79 (s, 3H), 1.04 (s, 9H), 0.27 (s, 3H), 0.24 $(\mathrm{s}, 3 \mathrm{H}) ;{ }^{13} \mathrm{C}$ NMR $\left(100 \mathrm{MHz}, \mathrm{CDCl}_{3}\right) \delta 170.9,170.6,169.7$, 169.3, 160.8, 155.1, 129.0, 118.9, 106.5, 105.4, 77.4, 76.3, 75.0, 71.5, 68.9, 62.6, 55.4, 25.9, 20.90, 20.86, 20.80, 20.6, 18.4, -4.0, -4.3. HRMS (ESI) calcd for $\mathrm{C}_{27} \mathrm{H}_{40} \mathrm{O}_{11} \mathrm{NaSi}, 591.2238(\mathrm{M}+\mathrm{Na})$, found $\mathrm{m} / \mathrm{z}, 591.2222$. 
To a solution of the acetyl ester $(291 \mathrm{mg}, 0.512 \mathrm{mmol})$ in THF $(10.3 \mathrm{~mL})$ and $\mathrm{H}_{2} \mathrm{O}(9.0 \mu \mathrm{L})$ were added $\mathrm{AcOH}(30 \mu \mathrm{L})$ and TBAF $\left(0.5 \mathrm{~mL}, 1 \mathrm{M}\right.$ solution in THF) at $0{ }^{\circ} \mathrm{C}$. After $18 \mathrm{~h}$, the 1 reaction mixture was diluted with EtOAc, and washed with brine,
$165.41,139.1,138.2,133.6,133.5,133.3,133.26,130.0,129.91$, $129.88,129.83,129.75,129.0,128.93,128.88,128.57,128.53$, 128.50, 128.40, 103.9, 94.6, 76.7, 74.5, 73.2, 72.2, 69.9, 63.2, 56.3, 51.67, 51.66. HRMS (ESI) calcd for $\mathrm{C}_{43} \mathrm{H}_{38} \mathrm{O}_{13} \mathrm{Na}$, $785.2210(\mathrm{M}+\mathrm{Na})$, found $m / z, 785.2209$.

\subsection{3. $(2 R, 3 R, 4 R, 5 S, 6 S)-2-((($ tert -}

Butoxycarbonyl)oxy)methyl)-6-(3,3,4-trimethoxy-6oxocyclohexa-1,4-dien-1-yl)tetrahydro-2H-pyran3,4,5-triyl tri-tert-butyl tricarbonate (11)

A solution of the siloxy ether of $\mathbf{4 a}(74 \mathrm{mg}, 0.0973 \mathrm{mmol})$ in $\mathrm{MeOH}(1.5 \mathrm{~mL})$ was submitted to catalytic hydrogenation in the presence of $5 \% \mathrm{Pd}-\mathrm{C}(10 \mathrm{~mol} \%)$. A crude product was acylated with $(\mathrm{Boc})_{2} \mathrm{O}(0.10 \mathrm{~mL})$ and TEA $(0.33 \mathrm{~mL})$ in THF $(2 \mathrm{~mL})$ to afford a Boc ester as a colorless oil $\left(54.5 \mathrm{mg}, 70 \%\right.$ yield): $[\alpha]_{\mathrm{D}}{ }^{21}$ $+7.4\left(c 1.00, \mathrm{CHCl}_{3}\right)$; IR (film) $1759 \mathrm{~cm}^{-1} ;{ }^{1} \mathrm{H}$ NMR $(400 \mathrm{MHz}$, $\left.\mathrm{CDCl}_{3}\right) \delta 7.30(\mathrm{~d}, J=8.4 \mathrm{~Hz}, 1 \mathrm{H}), 6.49(\mathrm{dd}, J=8.4,2.4 \mathrm{~Hz}, 1 \mathrm{H})$, $6.31(\mathrm{~d}, J=2.4 \mathrm{~Hz}, 1 \mathrm{H}), 5.13(\mathrm{t}, J=9.4 \mathrm{~Hz}, 1 \mathrm{H}), 5.06(\mathrm{t}, J=9.4$ $\mathrm{Hz}, 1 \mathrm{H}), 4.97$ (t, $J=9.4 \mathrm{~Hz}, 1 \mathrm{H}), 4.83$ (d, $J=9.4 \mathrm{~Hz}, 1 \mathrm{H}), 4.24$ $(\mathrm{dd}, J=12.0,5.4 \mathrm{~Hz}, 1 \mathrm{H}), 4.12(\mathrm{dd}, J=12.0,2.6 \mathrm{~Hz}, 1 \mathrm{H}), 3.79$ (ddd, $J=9.4,5.4,2.6 \mathrm{~Hz}, 1 \mathrm{H}), 3.73(\mathrm{~s}, 3 \mathrm{H}), 1.47$ (s, 9H), 1.45 (s, 9H), $1.43(\mathrm{~s}, 9 \mathrm{H}), 1.26(\mathrm{~s}, 9 \mathrm{H}), 1.01(\mathrm{~s}, 9 \mathrm{H}), 0.26(\mathrm{~s}, 3 \mathrm{H}), 0.23(\mathrm{~s}$, $3 \mathrm{H}) ;{ }^{13} \mathrm{C}$ NMR $\left(100 \mathrm{MHz}, \mathrm{CDCl}_{3}\right) \delta 160.7,155.3,153.3,152.6$, $152.2,152.0,149.4,146.9,106.1,105.1,85.3,83.1,82.6,82.2$, 77.8, 77.4, 77.2, 76.2, 73.8, 72.0, 65.2, 55.4, 27.86, 27.84, 27.78, 27.6, 26.0, 25.8, 21.2, 18.4, 14.3, -4.1, -4.3. HRMS (ESI) calcd for $\mathrm{C}_{39} \mathrm{H}_{64} \mathrm{O}_{15} \mathrm{NaSi}, 823.3912(\mathrm{M}+\mathrm{Na})$, found $\mathrm{m} / \mathrm{z}$ 823.3911.

A solution of the ester $(10 \mathrm{mg}, 0.0125 \mathrm{mmol}))$ and TBAF $(0.0125 \mathrm{~mL})$ in THF $(0.25 \mathrm{~mL})$ was kept at room temperature for $4 \mathrm{~h}$, to afford a phenol as colorless oil $(7.7 \mathrm{mg}, 90 \%):[\alpha]_{\mathrm{D}}{ }^{23}-16.0$ (c 1.00, $\mathrm{CHCl}_{3}$ ); IR (film) 3437, $1757 \mathrm{~cm}^{-1}$; ${ }^{1} \mathrm{H}$ NMR $(400 \mathrm{MHz}$, $\left.\mathrm{CDCl}_{3}\right) \delta 6.94(\mathrm{~s}, 1 \mathrm{H}), 6.90(\mathrm{~d}, J=8.5 \mathrm{~Hz}, 1 \mathrm{H}), 6.45(\mathrm{~d}, J=2.5$ $\mathrm{Hz}, 1 \mathrm{H}), 6.36(\mathrm{dd}, J=8.5,2.5 \mathrm{~Hz}, 1 \mathrm{H}), 5.12(\mathrm{t}, J=9.6 \mathrm{~Hz}, 1 \mathrm{H})$, $5.02(\mathrm{t}, J=9.6 \mathrm{~Hz}, 2 \mathrm{H}), 4.50(\mathrm{~d}, J=9.6 \mathrm{~Hz}, 1 \mathrm{H}), 4.37(\mathrm{dd}, J=$ $12.2,5.1 \mathrm{~Hz}, 1 \mathrm{H}), 4.18(\mathrm{dd}, J=12.2,2.5 \mathrm{~Hz}, 1 \mathrm{H}), 3.86(\mathrm{ddd}, J=$ 9.6, 5.1, 2.5 Hz, 1H), 3.73 (s, 3H), 1.47 (s, 9H), 1.45 (s, 9H), 1.44 $(\mathrm{s}, 9 \mathrm{H}), 1.24(\mathrm{~s}, 9 \mathrm{H}) ;{ }^{13} \mathrm{C}$ NMR $\left(100 \mathrm{MHz}, \mathrm{CDCl}_{3}\right) \delta 161.5$, $156.9,153.3,152.6,152.1,151.3,129.6,112.7,106.4,103.0$, 83.6, 82.9, 82.8, 80.7, 77.4, 76.6, 76.3, 73.3, 71.0, 64.3, 55.4, 28.6, 28.5, 28.4, 27.8, 27.7, 27.5. HRMS (ESI) calcd for $\mathrm{C}_{33} \mathrm{H}_{50} \mathrm{O}_{15} \mathrm{Na}, 709.3047(\mathrm{M}+\mathrm{Na})$, found $\mathrm{m} / \mathrm{z} 709.3025$.

Oxidation of the phenol $(7.50 \mathrm{mg}, 0.0109 \mathrm{mmol})$ in $\mathrm{MeOH}(1$ $\mathrm{mL})$ with $\mathrm{PhI}(\mathrm{OAc})_{2}(10 \mathrm{mg}, 0.0311 \mathrm{mmol})$ afford $\mathbf{1 1}$ as a yellow oil (5.9 mg, 73\%): $[\alpha]_{\mathrm{D}}{ }^{21}-23.5\left(c 1.00, \mathrm{CHCl}_{3}\right)$; IR (film) 1759, $1254 \mathrm{~cm}^{-1} ;{ }^{1} \mathrm{H}$ NMR $\left(400 \mathrm{MHz}, \mathrm{CDCl}_{3}\right) \delta 6.77(\mathrm{~s}, 1 \mathrm{H}), 5.61(\mathrm{~s}$, $1 \mathrm{H}), 5.10(\mathrm{t}, J=9.6 \mathrm{~Hz}, 1 \mathrm{H}), 4.90(\mathrm{t}, J=9.6 \mathrm{~Hz}, 1 \mathrm{H}), 4.85$ (t, $J=$ $9.6 \mathrm{~Hz}, 1 \mathrm{H}), 4.66(\mathrm{~d}, J=9.6 \mathrm{~Hz}, 1 \mathrm{H}), 4.27(\mathrm{dd}, J=12.1,5.4 \mathrm{~Hz}$, $1 \mathrm{H}), 4.13$ (dd, $J=12.1,2.4 \mathrm{~Hz}, 1 \mathrm{H}), 3.79$ (ddd, $J=9.6,5.4,2.4$ $\mathrm{Hz}, 1 \mathrm{H}), 3.78$ (s, 3H), 3.298 (s, 3H), 3.296 (s, 3H), 1.46 (s, 18H), $1.44(\mathrm{~s}, 9 \mathrm{H}), 1.37(\mathrm{~s}, 9 \mathrm{H}) ;{ }^{13} \mathrm{C}$ NMR $\left(100 \mathrm{MHz}, \mathrm{CDCl}_{3}\right) \delta 183.8$, 168.7, 153.3, 152.4, 152.20, 152.16, 138.9, 138.0, 104.1, 94.6, 83.2, 82.74, 82.67, 82.5, 77.0, 76.2, 74.9, 72.1, 71.5, 65.0, 56.3, 51.8, 51.7, 27.9, 27.81, 27.77, 27.7. HRMS (ESI) calcd for $\mathrm{C}_{35} \mathrm{H}_{54} \mathrm{O}_{17} \mathrm{Na}, 769.3259(\mathrm{M}+\mathrm{Na})$, found $\mathrm{m} / \mathrm{z} 769.3251$.

\subsection{Typical procedure for hydrogenation of $C$-glycosides}

To a solution of substrate $(20 \mathrm{mg})$ in solvent $(0.05 \mathrm{M})$ was added 5\% Pd-C (10 mol \%). After being stirred under $\mathrm{H}_{2}$ atmosphere at room temperature for $2 \sim 9 \mathrm{~h}$, the reaction mixture was filtered, and concentrated in vacuo. The resulting residue was purified by PTLC (hexane : EtOAc $=1 / 1 \sim 1 / 3$, two times developed) to give the corresponding products.

4.4.1. (R)-3,4,4-Trimethoxy-6-((2S,3S,4R, 5R,6R)3,4,5-tris(benzyloxy)-6-(methoxymethyl)tetrahydro2H-pyran-2-yl)cyclohex-2-en-1-one (7a) and (S)- 
3,4,4-trime thoxy-6-((2S,3S,4R,5R,6R)-3,4,5tris(benzyloxy)-6-(methoxymethyl)tetrahydro- $2 \mathrm{H}$ pyran-2-yl)cyclohex-2-en-1-one (7b)

7a: $[\alpha]_{\mathrm{D}}{ }^{24}-56.5\left(c 1.00, \mathrm{CHCl}_{3}\right)$; IR (film) $1652,1616 \mathrm{~cm}^{-1} ;{ }^{1} \mathrm{H}$ (13a), (2R,3R,4R,5S,6S)-2-((Benzoyloxy)methyl)-6((S)-4,5,5-trimethoxy-2-oxocyclohex-3-en-1yl)tetrahydro-2H-pyran-3,4,5-triyl tribenzoate $(13 \mathrm{~b})$ and $(2 R, 3 R, 4 R, 5 S, 6 S)-2$ -

((Benzoyloxy)methyl)-6-(2-hydroxy-4,5dimethoxyphenyl)tetrahydro-2H-pyran-3,4,5-triyl tribenzoate (15)

13a: $[\alpha]_{\mathrm{D}}^{23}-51.4\left(c 1.00, \mathrm{CHCl}_{3}\right)$; IR (film) $2947,1730 \mathrm{~cm}^{-1}$; ${ }^{1} \mathrm{H}$ NMR $\left(400 \mathrm{MHz}, \mathrm{CDCl}_{3}\right) \delta 8.02(\mathrm{dd}, J=8.3,1.0 \mathrm{~Hz}, 2 \mathrm{H})$, $7.90(\mathrm{dd}, J=8.3,1.0 \mathrm{~Hz}, 2 \mathrm{H}), 7.84(\mathrm{dd}, J=8.3,1.5 \mathrm{~Hz}, 2 \mathrm{H})$, $7.79(\mathrm{dd}, J=8.3,1.5 \mathrm{~Hz}, 2 \mathrm{H}), 7.56-7.24(\mathrm{~m}, 12 \mathrm{H}), 5.87(\mathrm{t}, J=$ $9.7 \mathrm{~Hz}, 1 \mathrm{H}), 5.75(\mathrm{t}, J=9.7 \mathrm{~Hz}, 1 \mathrm{H}), 5.64(\mathrm{t}, J=9.7 \mathrm{~Hz}, 1 \mathrm{H})$, $4.98(\mathrm{~s}, 1 \mathrm{H}), 4.68(\mathrm{dd}, J=9.7,2.2 \mathrm{~Hz}, 1 \mathrm{H}), 4.58(\mathrm{dd}, J=12.0$, $2.8 \mathrm{~Hz}, 1 \mathrm{H}), 4.42$ (dd, $J=12.0,5.6 \mathrm{~Hz}, 1 \mathrm{H}), 4.17$ (ddd, $J=9.8$, 5.6, $2.8 \mathrm{~Hz}, 1 \mathrm{H}), 3.47$ (s, 3H), $3.36(\mathrm{~s}, 3 \mathrm{H}), 3.19$ (s, 3H), 3.13 (ddd, $J=12.5,4.9,2.4 \mathrm{~Hz}, 1 \mathrm{H}), 2.56(\mathrm{dd}, J=12.5,4.9 \mathrm{~Hz}, 1 \mathrm{H})$, $2.10(\mathrm{t}, J=12.5 \mathrm{~Hz}, 1 \mathrm{H}) ;{ }^{13} \mathrm{C}$ NMR $\left(100 \mathrm{MHz}, \mathrm{CDCl}_{3}\right) \delta 195.0$, $172.7,166.3,165.9,165.4,165.1,133.5,133.4,133.2,129.9$, $129.83,129.79,129.77,129.03,128.96,128.9,128.63,128.56$, 128.48, 128.45, 128.4, 128.3, 103.3, 97.4, 76.7, 75.0, 70.0, 69.8, 63.6, 56.0, 51.1, 49.1, 45.0, 31.8. HRMS (ESI) calcd for $\mathrm{C}_{43} \mathrm{H}_{40} \mathrm{O}_{13} \mathrm{Na}$, 787.2367 (M+Na), found $\mathrm{m} / z$ 787.2347. 13b: $[\alpha]_{\mathrm{D}}{ }^{22}$ $+45.4\left(c\right.$ 1.00, $\left.\mathrm{CHCl}_{3}\right)$; IR (film) 2942, $1732 \mathrm{~cm}^{-1} ;{ }^{1} \mathrm{H}$ NMR (400 $\left.\mathrm{MHz}, \mathrm{CDCl}_{3}\right) \delta 8.00(\mathrm{dd}, J=8.3,1.1 \mathrm{~Hz}, 2 \mathrm{H}), 7.93(\mathrm{dd}, J=8.4$, $1.2 \mathrm{~Hz}, 2 \mathrm{H}), 7.90(\mathrm{dd}, J=8.2,1.2 \mathrm{~Hz}, 2 \mathrm{H}), 7.80(\mathrm{dd}, J=8.2,1.2$ $\mathrm{Hz}, 2 \mathrm{H}), 7.56-7.24(\mathrm{~m}, 12 \mathrm{H}), 5.95$ (t, $J=9.8 \mathrm{~Hz}, 1 \mathrm{H}), 5.63$ (t, $J=$ $9.8 \mathrm{~Hz}, 1 \mathrm{H}), 5.55(\mathrm{t}, J=9.8 \mathrm{~Hz}, 1 \mathrm{H}), 5.32(\mathrm{~s}, 1 \mathrm{H}), 4.79(\mathrm{dd}, J=$ $9.8,1.1 \mathrm{~Hz}, 1 \mathrm{H}), 4.51(\mathrm{dd}, J=12.1,3.1 \mathrm{~Hz}, 1 \mathrm{H}), 4.39(\mathrm{dd}, J=$ $12.1,4.9 \mathrm{~Hz}, 1 \mathrm{H}), 4.15$ (ddd, $J=9.8,4.9,3.1 \mathrm{~Hz}, 1 \mathrm{H}), 3.70$ (s, $3 \mathrm{H}), 3.41(\mathrm{~s}, 3 \mathrm{H}), 3.15(\mathrm{~s}, 3 \mathrm{H}), 2.67(\mathrm{~m}, 2 \mathrm{H}), 2.22(\mathrm{t}, J=13.5 \mathrm{~Hz}$, $1 \mathrm{H}) ;{ }^{13} \mathrm{C}$ NMR $\left(100 \mathrm{MHz}, \mathrm{CDCl}_{3}\right) \delta 196.5,173.3,166.2,165.9$, 165.6, 165.4, 133.6, 133.5, 133.3, 133.1, 130.03, 129.97, 129.91, 129.89, 129.8, 129.1, 129.0, 128.6, 128.54, 128.46, 128.4, 103.7, 97.6, 77.5, 77.4, 77.2, 76.8, 76.1, 75.6, 75.0, 69.9, 69.1, 63.1, 56.2, 50.9, 49.4, 29.6. HRMS calcd for $\mathrm{C}_{43} \mathrm{H}_{40} \mathrm{O}_{13} \mathrm{Na}, 787.2367$ $(\mathrm{M}+\mathrm{Na})$, found $m / z$ 787.2350. 15: $[\alpha]_{\mathrm{D}}{ }^{24}-48.7$ (c 1.00, $\left.\mathrm{CHCl}_{3}\right)$; IR (film) 3447, $1730 \mathrm{~cm}^{-1} ;{ }^{1} \mathrm{H}$ NMR (400 MHz, $\left.\mathrm{CDCl}_{3}\right) \delta 8.09$ $(\mathrm{dd}, J=8.3,1.3 \mathrm{~Hz}, 2 \mathrm{H}), 7.93(\mathrm{dd}, J=8.4,1.2 \mathrm{~Hz}, 2 \mathrm{H}), 7.83(\mathrm{dd}$, $J=8.1,1.3 \mathrm{~Hz}, 2 \mathrm{H}), 7.82(\mathrm{dd}, J=8.3,1.3 \mathrm{~Hz}, 2 \mathrm{H}), 7.61-7.25(\mathrm{~m}$, $12 \mathrm{H}), 6.92(\mathrm{~s}, 1 \mathrm{H}), 6.48(\mathrm{~s}, 1 \mathrm{H}), 6.46(\mathrm{~s}, 1 \mathrm{H}), 6.04(\mathrm{t}, J=9.8 \mathrm{~Hz}$, $1 \mathrm{H}), 5.88(\mathrm{t}, J=9.8 \mathrm{~Hz}, 1 \mathrm{H}), 5.83(\mathrm{t}, J=9.8 \mathrm{~Hz}, 1 \mathrm{H}), 4.90(\mathrm{~d}, J=$ $9.8 \mathrm{~Hz}, 1 \mathrm{H}), 4.66(\mathrm{dd}, J=12.4,2.7 \mathrm{~Hz}, 1 \mathrm{H}), 4.58(\mathrm{dd}, J=12.4$, $3.9 \mathrm{~Hz}, 1 \mathrm{H}), 4.32(\mathrm{ddd}, J=9.8,3.9,2.7 \mathrm{~Hz}, 1 \mathrm{H}), 3.78(\mathrm{~s}, 3 \mathrm{H})$, $3.37(\mathrm{~s}, 3 \mathrm{H}) ;{ }^{13} \mathrm{C}$ NMR $\left(100 \mathrm{MHz}, \mathrm{CDCl}_{3}\right) \delta 166.3,166.1,165.2$, 164.6, 150.5, 149.9, 142.3, 133.7, 133.5, 133.42, 133.40, 130.01, $129.95,129.9,129.8,129.7,129.5,129.1,128.9,128.8,128.7$, $128.6,128.5,128.4,111.6,110.7,102.3,80.8,76.9,74.0,71.6$, 69.1, 62.6, 56.1, 55.9. HRMS (ESI) calcd for $\mathrm{C}_{42} \mathrm{H}_{37} \mathrm{O}_{12} \mathrm{Na}$, 733.2285(M+Na), found $\mathrm{m} / \mathrm{z}$ 733.2270.

\subsection{4. $(2 R, 3 R, 4 R, 5 S, 6 S)-2-((($ tert -}

Butoxycarbonyl)oxy)methyl $)-6-((R)-4,5,5-$ trimethoxy-2-oxocyclohex-3-en-1-yl)tetrahydro-2Hpyran-3,4,5-triyl tri-tert-butyl tricarbonate (14a) and $(2 R, 3 R, 4 R, 5 S, 6 S)-2-((($ tert -

Butoxycarbonyl)oxy)methyl)-6-((S)-4,5,5trimethoxy-2-oxocyclohex-3-en-1-yl)tetrahydro-2Hpyran-3,4,5-triyl tri-tert-butyl tricarbonate (14b)

14a: $[\alpha]_{\mathrm{D}}{ }^{21}-61.7\left(c\right.$ 1.00, $\mathrm{CHCl}_{3}$ ); IR (film) $1759 \mathrm{~cm}^{-1} ;{ }^{1} \mathrm{H}$ NMR (400 MHz, $\left.\mathrm{CDCl}_{3}\right) \delta 5.29(\mathrm{~s}, 1 \mathrm{H}), 5.25(\mathrm{t}, J=9.6 \mathrm{~Hz}, 1 \mathrm{H})$, $4.98(\mathrm{t}, J=9.6 \mathrm{~Hz}, 1 \mathrm{H}), 4.77(\mathrm{t}, J=9.6 \mathrm{~Hz}, 1 \mathrm{H}), 4.20(\mathrm{dd}, J=$ $12.0,6.5 \mathrm{~Hz}, 1 \mathrm{H}), 4.10(\mathrm{dd}, J=12.0,2.7 \mathrm{~Hz}, 1 \mathrm{H}), 4.03(\mathrm{dd}, J=$ 9.6, $2.2 \mathrm{~Hz}, 1 \mathrm{H}), 3.71(\mathrm{~s}, 3 \mathrm{H}), 3.39(\mathrm{~s}, 3 \mathrm{H}), 3.23(\mathrm{~s}, 3 \mathrm{H}), 3.00$ (ddd, $J=12.7,4.7,2.2 \mathrm{~Hz}, 1 \mathrm{H}), 2.40(\mathrm{dd}, J=12.6,4.7 \mathrm{~Hz}, 1 \mathrm{H})$, 1.44 (dd, $J=12.6,12.7 \mathrm{~Hz}, 1 \mathrm{H}), 1.47$ (s, 3H), 1.45 (s, 3H), 1.44 (s, 3H), $1.41(\mathrm{~s}, 3 \mathrm{H}) ;{ }^{13} \mathrm{C} \mathrm{NMR}\left(100 \mathrm{MHz}, \mathrm{CDCl}_{3}\right) \delta 195.1$, $172.2,153.2,152.5,152.2,152.1,103.9,97.5,83.1,82.7,82.54$, 
82.52, 77.6, 77.0, 76.2, 72.4, 71.9, 65.5, 56.1, 51.1, 49.2, 44.0, 32.8, 27.9, 27.84, 27.78. HRMS calcd for $\mathrm{C}_{35} \mathrm{H}_{56} \mathrm{O}_{17} \mathrm{Na}, 771.3415$ $(\mathrm{M}+\mathrm{Na})$, found $m / z$ 771.3397. 14b: $[\alpha]_{\mathrm{D}}{ }^{23}+10.0\left(c 1.00, \mathrm{CHCl}_{3}\right)$;
IR (film) $1759 \mathrm{~cm}^{-1} ;{ }^{1} \mathrm{H}$ NMR (400 MHz, $\left.\mathrm{CDCl}_{3}\right) \delta 5.36(\mathrm{~s}, 1 \mathrm{H})$, $5.03(\mathrm{t}, J=9.8 \mathrm{~Hz}, 1 \mathrm{H}), 4.83(\mathrm{t}, J=9.8 \mathrm{~Hz}, 2 \mathrm{H}), 4.39(\mathrm{dd}, J=$ $9.8,1.5 \mathrm{~Hz}, 1 \mathrm{H}), 4.23(\mathrm{dd}, J=12.1,4.2 \mathrm{~Hz}, 1 \mathrm{H}), 4.05(\mathrm{dd}, J=$ 12.1, $2.6 \mathrm{~Hz}, 1 \mathrm{H}), 3.74$ (s, 3H), 3.67 (ddd, $J=9.8,4.2,2.6 \mathrm{~Hz}$, $1 \mathrm{H}), 3.40(\mathrm{~s}, 3 \mathrm{H}), 3.19(\mathrm{~s}, 3 \mathrm{H}), 2.67(\mathrm{ddd}, J=12.7,4.5,1.5 \mathrm{~Hz}$, $1 \mathrm{H}), 2.56(\mathrm{dd}, J=12.7,4.5 \mathrm{~Hz}, 1 \mathrm{H}), 2.09(\mathrm{t}, J=12.7 \mathrm{~Hz}, 1 \mathrm{H})$, 1.455 (s, 9H), 1.448 (s, 18H), 1.445 (s, 9H); ${ }^{13} \mathrm{C} \mathrm{NMR} \mathrm{(100} \mathrm{MHz,}$ $\left.\mathrm{CDCl}_{3}\right) \delta 196.0,173.0,153.3,152.6,152.4,152.1,103.8,97.6$, 83.0, 82.5, 82.3, 75.8, 74.9, 71.5, 71.0, 64.5, 56.2, 50.9, 49.2, 42.9, 29.9, 27.90, 27.86, 27.8, 27.7. HRMS (ESI) calcd for $\mathrm{C}_{35} \mathrm{H}_{56} \mathrm{O}_{17} \mathrm{Na}, 771.3415(\mathrm{M}+\mathrm{Na})$, found $\mathrm{m} / z$, 771.3433 .

\subsection{Computational studies}

Theoretical caluculations were carried out using MOE (Molecular Operating Environment, ver. moe 2012. 1001) with force field (MMFF94x) ${ }^{20}$. Low Mode MD (initial temperature = $400 \mathrm{~K}$ ) implemented in MOE was used for the conformational approximations.

\section{Acknowledgments}

This study was supported by financial contributions from the Scientific Research C, MEXT and MEXT-Supported Programs for the Strategic Research Foundation at Private Universities, 2009-2013 and 2012-2016.

\section{References and notes}

1. (a) Iguchi, M.; Nishiyama, A.; Terada, Y.; Yamamura, S. Tetrahedron Lett. 1977, 51, 4511-4514; (b) Yamamura, S. in The Chemistry of Phenols; Part 2 (Ed. Z. Rappoport), 2003, Ch. 17, 1153-1346 (Wiley: Chichester); (c) Yamamura, S. (2009). Oxidation of Phenols. In PATAI'S Chemistry of Functional Groups. John Wiley \& Sons, Ltd.

2. (a) Hsu, D.-S.; Liao, C.-C. Org. Lett. 2007, 9, 4563-4565; (b) Kurti, L.; Szilagyi, L.; Antus, S.; Nogradi, M. Eur. J. Org. Chem. 1999, 10, 2579-2581.

3. (a) Büchi, G.; Chu, P.-S. J. Am. Chem. Soc. 1981, 103, 27182721; (b) Tamura, Y.; Yakura, T.; Haruta, J.; Kita, Y. J. Org. Chem. 1987, 52, 3927-3930. search. Solvent effects were included by Distance or Born
4. (a)Dolson, M. G.; Swenton, S. S. J. Org. Chem. 1981, 46, 177179; (b) Deffieux, D.; Fabre, I.; Courseille, C.; Quideau, S. J. Org. Chem. 2002, 67, 4458-4465.

5. (a) Chiba, K.; M. Tada. J. Chem. Soc., Chem. Commun. 1994, 2485-2486; (b) Chiba, K.; Arakawa, T.; M. Tada. J. Chem. Soc., Perkin Trans. 1 1998, 2939-2942; (c) Banfield, S. C.; Kerr, M. A. Can. J. Chem 2004, 82, 131-138.

6. Deajardins, S; Maertens, G; Canesi, S. Org. Lett. 2014, 16, 4928-4931.

7. Ogamino, T.; Obata, R.; Nishiyama, S. Tetrahedron Lett., 2006, 47, 727-731.

8. Kubo, H.; Matsui, K.; Saitoh, T.; Nishiyama, S. Tetrahedron 2014, 70, 6392-6397.

9. Luo, S.-Y.; Jang, Y.-J.; Liu, J.-Y.; Chu, C.-S.; Liao, C.-C.; Hung S.-C. Angew. Chem. Int. Ed. 2008, 47, 8082-8085.

10. Bosset, C.; Coffinier, R.; Peixoto, P. A.; El Assal, M.; Miqueu, K.; Sotiropoulos, J.-M.; Pouységu, L.; Quideau, S. Angew. Chem. Int. Ed. 2014, 53, 1-6.

11. An example, see: Saitoh, T.; Suzuki, E.; Takasugi, A.; Obata, R.; Ishikawa, Y.; Umezawa, K.; Nishiyama, S. Bioorg. Med. Chem. Lett. 2009, 19, 5383-5386.

12. (a) Schmidt, R. R.; Michel, M. Angew. Chem. Int. Ed. Engl. 1980, 19, 731-732. (b) Mahling, J.-A.; Schmidt, R. R. Synthesis, 1993, 3, 325-328.

13. Hanzner, H.; Palmacci, E. R.; Seeberger, P. H. Org. Lett. 2002, 4, 2965-2967.

14. Matsumoto, T.; Hosoya, T.; Suzuki, K. Synlett, 1991, 709-711.

15. Sumi, T.; Saitoh, T.; Natsui, K.; Yamamoto, T.; Atobe, M.; Einaga, Y.; Nishiyama, S. Angew. Chem. Int. Ed. 2012, 51, 54435446.

16.

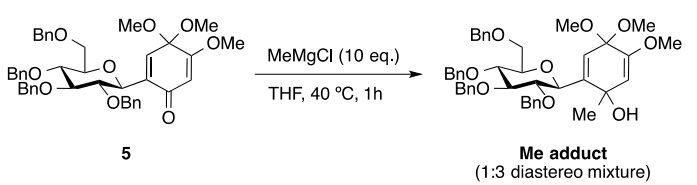

1,2-Addition reaction of $\mathbf{5}$ with Grignard and alkyl lithium nucleophiles provided the corresponding adducts in low to moderate yields with low diastereoselectivities. Optimized result was obtained in the reaction with $\mathrm{MeMgCl} / \mathrm{THF} 40{ }^{\circ} \mathrm{C}$, which afforded the product in 56\% yield with 1:3 diastereoselection. Contribution of reaction temperature indicated the steric hindrance of the substrate, which seemed to impede smooth attack of the reagent to the reactive point.

17. A good example for diastereoselective 1,4-addition to acyclic enone carrying glycosyl moiety; Totani, K.; Nagatsuka, T.; Yamaguchi, S.; Takao, K.; Ohba, S.; Tadano, K. J. Org. Chem. 2001, 66, 5965-5975.

18. Yamanoi, T.; Fujioka, A.; Inazu, T. Bull. Chem. Soc. Jpn. 1994, 67, 1488-1491.

19. Palmacci, E. R.; Seeberger, P. H. Org. Lett. 2001, 3, 1547-1550.

20. Halgren, T. A. J. Comp. Chem. 1996, 17, 490-519. 


\section{Graphical Abstract}

To create your abstract, type over the instructions in the template box below.

Fonts or abstract dimensions should not be changed or altered.

\section{Asymmetric induction in cyclohexadienones} carrying $\alpha$-D-glucopyranosyl moiety

Leave this area blank for abstract info.

Saki Yajima, Tsuyoshi Saitoh, Kohei Kawa, Kensuke Nakamura, Hiroshi Nagase, Yasuaki Einaga, Shigeru Nishiyama

International Institute for Integrative Sleep Medicine, Tsukuba University, Tennodai 1-1-1, Tsukuba 305-8575, Japan

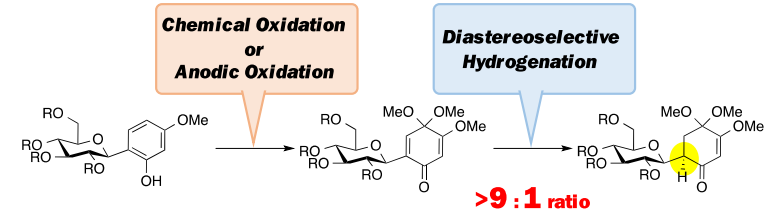

